\title{
Nodular Sclerosing Hodgkin Lymphoma Combined with Disseminated Talaromyces marneffei Infection: A Case Report
}

\author{
Zhenming Yang ${ }^{1,2}$ \\ Wen Zeng ${ }^{2}$ \\ Ye Qiu ${ }^{3}$ \\ Guangnan $\mathrm{Liu}^{4}$ \\ Jianquan Zhang'
}

'Department of Respiratory and Critical Medicine, The Eighth Affiliated Hospital, Sun Yat-sen University, Shenzhen, Guangdong, 518000, People's Republic of China; ${ }^{2}$ Department of Respiratory Medicine, The First Affiliated Hospital of Guangxi Medical University, Nanning, Guangxi, 53002I, People's Republic of China; ${ }^{3}$ Department of Comprehensive Internal Medicine, The Affiliated Tumor Hospital of Guangxi Medical University, Nanning, Guangxi, 53002I, People's Republic of China; ${ }^{4}$ Department of Respiratory Medicine, The Second Affiliated Hospital of Guangxi Medical University, Nanning, Guangxi, 530005, People's Republic of China
Correspondence: Jianquan Zhang Department of Respiratory and Critical Medicine, The Eighth Affiliated Hospital Sun Yat-sen University, No. 3025,

Shennan Middle Road, Shenzhen, 5I8000, Guangdong, People's Republic of China

Tel +8613978123845

$\mathrm{Fax}+86755-23482484$

Email jqzhang2002@126.com
Background: Talaromyces marneffei (TM) is the only temperature-biphasic pathogen among Penicillium spp. that causes talaromycosis marneffei (TSM). Clinical manifestations include fever, cough, expectoration, superficial and deep lymph node enlargement, hepatosplenomegaly, subcutaneous nodules, and bone and joint infections. Cases of TSM in Hodgkin lymphoma (HL) patients are uncommon. The clinical manifestations and imaging findings are similar in TSM and HL, which make it difficult for clinicians to distinguish between TSM and HL. Both diseases can present with symptoms, can involve the blood or the respiratory system and can include other symptoms. We report a rare case of HIVnegative nodular sclerosing Hodgkin lymphoma (NSHL) combined with T. marneffei infection to improve clinical knowledge.

Case Presentation: The patient was a 51-year-old man who presented with a 1-month history of cough, expectoration, intermittent fever in the afternoon and night, cervical lymph node enlargement, diabetes and previous lung surgery. He had markedly elevated serum inflammatory markers and moderate diffuse lung dysfunction. Chest computed tomography (CT) showed diffuse nodular lesions in both lungs with mediastinal lymph node enlargement. The patient did not respond to antibacterial and diagnostic antituberculosis therapy. After lymph node biopsy and lung culture, we obtained a definite diagnosis of NSLH with T. marneffei infection and administered antifungal therapy. His symptoms improved, and he was discharged for further treatment. Unfortunately, he died of Salmonella sepsis 7 months later.

Conclusion: It is rare for NSLH patients to be infected with T. marneffei. Both diseases can present with fever, lymphadenopathy, and hepatosplenomegaly and involve the blood and respiratory system or can cause other symptoms. Clinically, a misdiagnosis or missed diagnosis may occur. A multisite biopsy or culture should be performed to make a definitive diagnosis. Early antifungal therapy combined with standard chemotherapy can achieve satisfactory clinical efficacy.

Keywords: Talaromyces marneffei, nodular sclerosing Hodgkin lymphoma, identification, treatment

\section{Introduction}

Talaromyces marneffei (TM, formerly known as Penicillium marneffei) is the only temperature-biphasic pathogen among the Penicillium spp. that causes talaromycosis marneffei (TSM), a rare deep fungal infection with systemic spread. HIV-negative hosts with underlying diseases are also susceptible to T. marneffei. ${ }^{1}$ Clinical manifestations are fever, cough, sputum, superficial and deep lymphadenopathy, hepatosplenomegaly, subcutaneous nodules, and bone and joint infections. Infection with TSM in HIV-negative 
hosts within the blood is mostly reported in individual case reports. Nodular sclerosing Hodgkin lymphoma (NSHL) is the most common subtype of Hodgkin lymphoma and can present with fever, multiple painless enlargements of lymph nodes, systemic symptoms and/or multisystem damage. ${ }^{2,3}$ Clinicians have difficulty distinguishing NSHL and TSM due to their similar clinical characteristics. The presence of both diseases makes the illness more complex and difficult to treat. Here, we report a rare case of HIV-negative NSHL combined with T. marneffei infection to improve clinical knowledge.

\section{Case Presentation}

The patient was a 51-year-old male from southern China who was hospitalized with cough, expectoration, intermittent afternoon and nighttime fever, cervical lymph node enlargement for one month, fatigue, night sweats, shortness of breath, and emaciation. He had a history of diabetes for more than 1 year and had good blood glucose control with insulin therapy. He had been a smoker for 6 years and had a previous excision of a left lung abscess but denied a history of contact with bamboo rats. On admission, the patient had a fever (highest temperature $40.3^{\circ} \mathrm{C}$ ). The patient had multiple enlarged lymph nodes in the neck and right supraclavicular fossa, the largest of which was $2 \mathrm{~cm} \times 1 \mathrm{~cm}$ in size in the right supraclavicular fossa. It was hard and fixed and had a clear boundary with the surrounding area without tenderness. There were subcutaneous nodules under the xiphoid process $(2 \mathrm{~cm} \times 2 \mathrm{~cm})$ and on the outside of his right thigh $(4 \mathrm{~cm} \times 4 \mathrm{~cm})$, which were hard and fixed without swelling and tenderness. The liver and spleen were not palpable under his ribs. His serum white blood cell (WBC) count, neutrophil ratio (NEUT), lymphocyte ratio, hemoglobin level, platelet count (PLT), procalcitonin (PCT) level, C-reactive protein (CRP) level, and erythrocyte sedimentation rate (ESR) were $24.85 \times 10^{9} /$ $\mathrm{L}, 87.30 \%, 6.50 \%, 79.8 \mathrm{~g} / \mathrm{L}, 410.00 \times 109 / \mathrm{L}, 1.47 \quad \mathrm{ng} /$ $\mathrm{mL}, 167.78 \mathrm{mg} / \mathrm{L}$, and $139 \mathrm{~mm} / \mathrm{h}$, respectively. His serum potassium, albumin, globulin, lactate dehydrogenase (LDH), creatinine, immunoglobulinG, complement C3, and complement C4 levels were $3.2 \mathrm{mmol} / \mathrm{L}, 20.1 \mathrm{~g} / \mathrm{L}$, $49.5 \mathrm{~g} / \mathrm{L}, 197 \mathrm{U} / \mathrm{L}, 80 \mu \mathrm{mol} / \mathrm{L}, 21.55 \mathrm{~g} / \mathrm{L}, 1.08 \mathrm{~g} / \mathrm{L}$, and $0.26 \mathrm{~g} / \mathrm{L}$, respectively. EB virus shell antigen (EBVCAIGA) and EB virus core antigen (EBNA1-IGA) were negative. No abnormal monoclonal bands were found in serum-immobilized electrophoresis. Tumor markers, immunoglobulinA, immunoglobulinM and total $\mathrm{T}$ lymphocytes were in the normal ranges. The blood and sputum cultures were negative, and the patient was HIV- negative. Lung function showed normal lung ventilation, a negative diastolic test, and moderate diffuse dysfunction. Chest CT showed diffuse nodular lesions in both lungs with mediastinal lymph node enlargement (Figure 1A and $\mathrm{B})$.

He was suspected of having bacterial pneumonia and tuberculosis. After receiving piperacillin sodium tazobactam sodium + azithromycin, imipenem, moxifloxacin + cefathiamidine, and diagnostic anti-tuberculosis treatment for 2 weeks (isoniazid $0.3 \mathrm{~g}$ qd + rifampicin $0.45 \mathrm{~g}$ qd + ethambutol 0.75 g qd + Pyrazinamide $1.0 \mathrm{~g} \mathrm{qd}$ ), the patient's condition deteriorated, and he had recurrent hyperpyrexia. His hemoglobin, albumin and other indicators then decreased significantly (hemoglobin $56.1 \mathrm{~g} / \mathrm{L}$, albumin $15.9 \mathrm{~g} / \mathrm{L}$ ). At that time, the competent doctor completed a right supraclavicular lymph node and bone marrow puncture biopsy after considering the possibility of the patient having a malignant tumor and other diseases. A background of complex inflammatory cells scattered with heterogeneous Hodgkin-like large cells was found in the histological examination of the lymph node. Immunohistochemistry revealed that the heteromorphic large cells were $\mathrm{CD} 30(+), \operatorname{CD} 20(-), \operatorname{PAX} 5( \pm)$, and CD3(-), and the in situ hybridization results were EBER (-). Bone marrow biopsy in this patient suggested mild hyperplasia of bone marrow hematopoietic tissue, with a small number of naive plasma cells present away from the trabecular bone (no significant atypia) and local fibrous hyperplasia with plasma cell hyperplasia (approximately 20\%). Immunohistochemistry results showed MPO $(+)$, CD34 $(+)$, and CD38 $(+)$, and Masson staining showed mild fibrous hyperplasia (Figure 2). The patient was diagnosed with NSHL (Stage II, Group B) (Figure 3). The patient refused chemotherapy, radiotherapy and targeted therapy. After treatment with prednisone acetate tablets $10 \mathrm{mg}$ three times a day, the patient's cervical lymph nodes decreased in size, but the patient's condition progressed, with continued hyperpyrexia. A repeat chest CT showed an increase in both of the lung lesions on January 15, 2018 (Figure 1C and D). The clinicians then considered that the patient may have other diseases in addition to NSHL. A bronchoscopic examination showed that the trachea and the walls of the left and right main bronchus were scattered with small nodules, and the nodules were partially fused near the carina (Figure 4A-C). Histopathology of nodules of lung mucosa at the right main bronchial orifice showed local chronic inflammatory disease accompanied by acute inflammation and small foci of suppurative necrosis. T. marneffei was found on the lung tissue culture (Figure 5A and B). Prednisone acetate tablets and antibacterial drugs were 


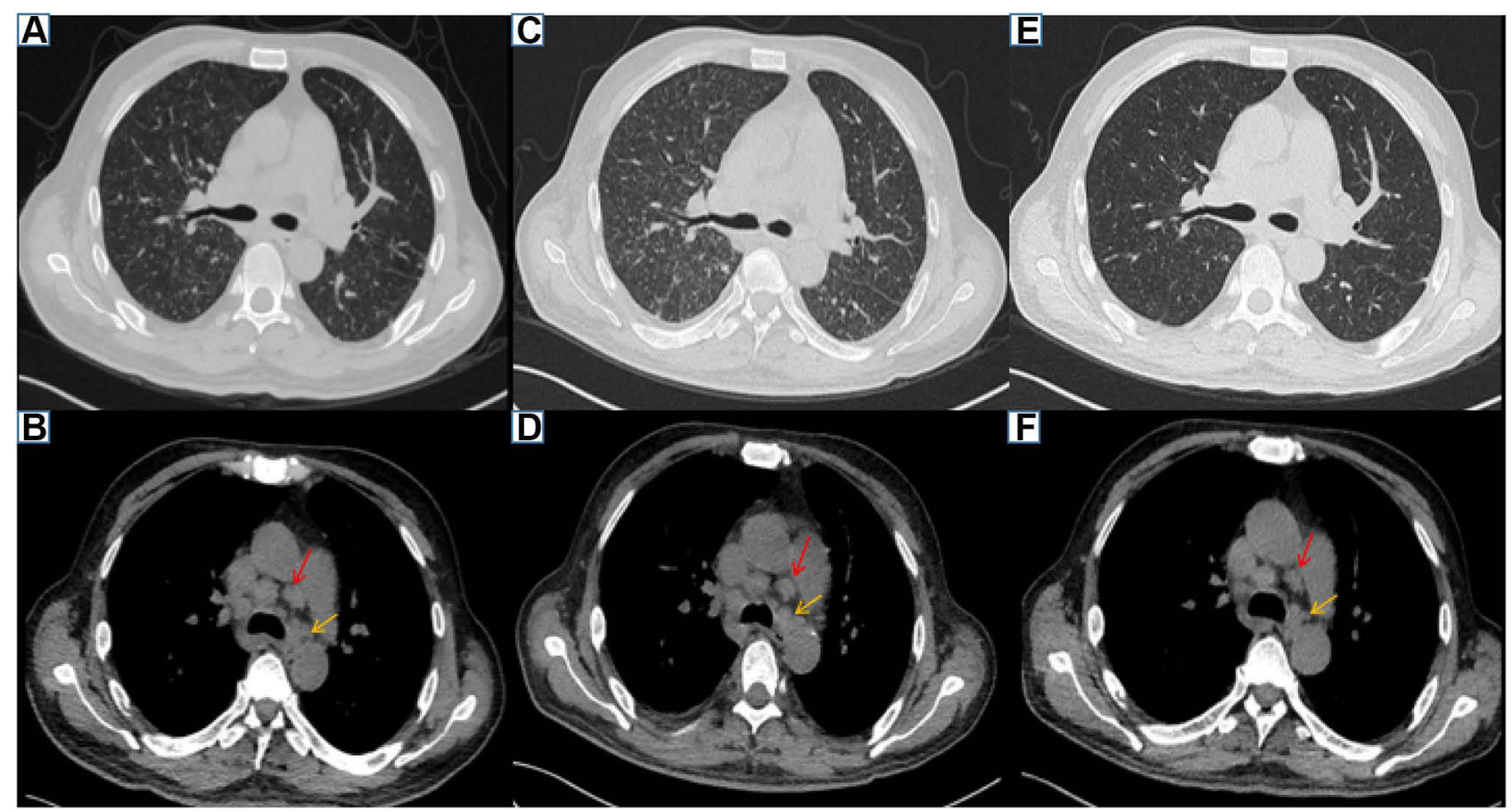

Figure I Chest CT (A-F). (A and B) Diffuse nodules in the lungs and multiple paratracheal lymph node enlargements on January I, 20I8. (C and D) The diffuse nodular shadows in the lungs were significantly increased, multiple lymph nodes around the trachea were enlarged and were unchanged compared with those in (A and $\mathbf{B}$ ), and a small amount of pleural effusion was observed on January 15, 20I8. (E and F) The diffuse nodules in the lungs were decreased in size, and multiple lymph nodes around the trachea had decreased in size on February 5, 2018. (red and yellow arrows indicate two enlarged lymph nodes).

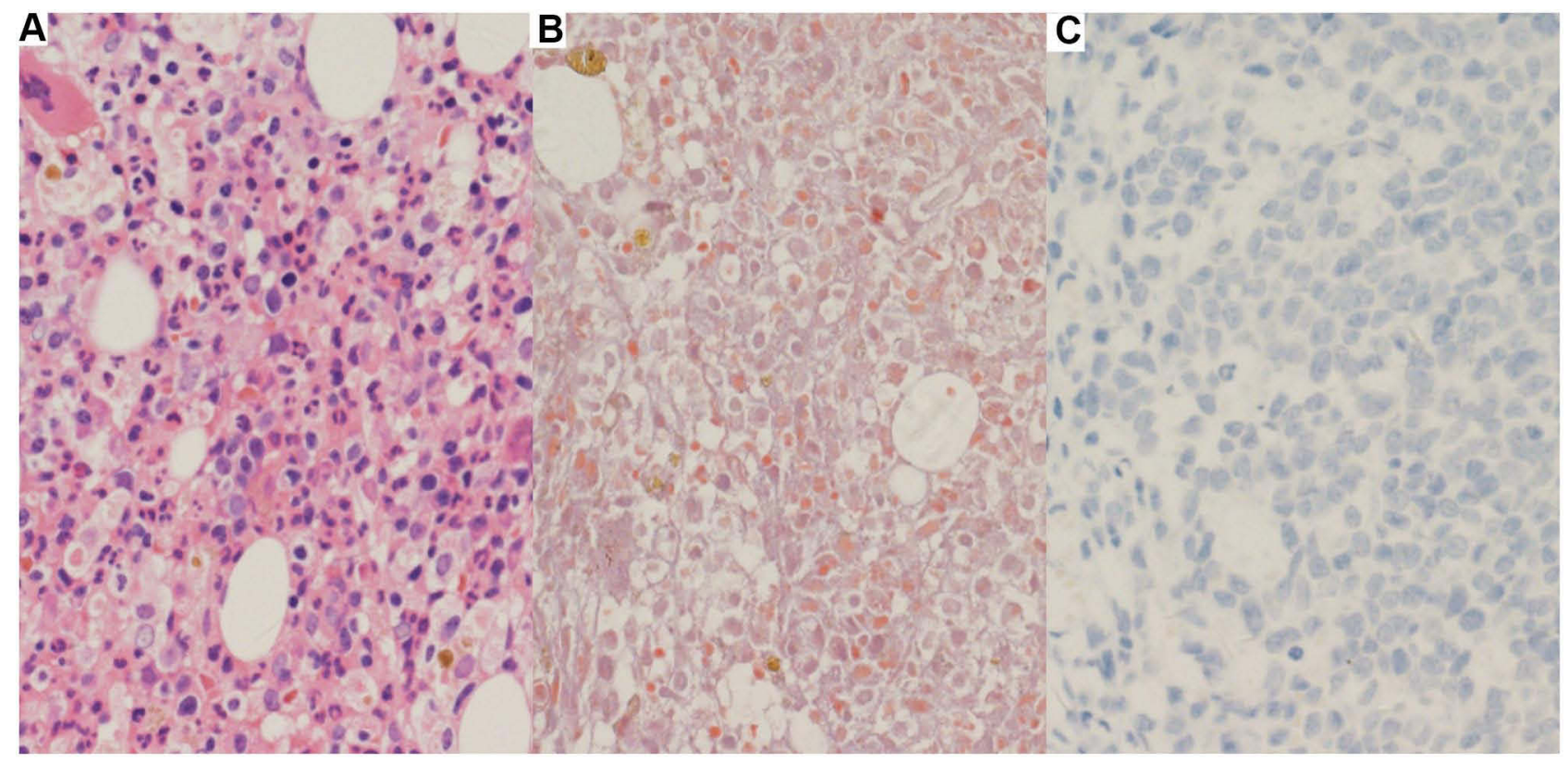

Figure 2 Bone marrow biopsy. (A-C) A small number of naive plasma cells were present away from the trabecular bone (no significant atypia), along with local fibrous hyperplasia with plasma cell hyperplasia (approximately $20 \%$ ). (A) HE, $\times 400$. (B) Masson staining (mild fibrous hyperplasia), $\times 400$. (C) MPO (+), $\times 200$.

discontinued on January 18, 2018. The patient's temperature returned to normal, the patient had no cough or expectoration, and the subcutaneous nodules decreased after antifungal therapy (after 14 days of intravenous amphotericin B liposome, the patient was switched to 6 days of intravenous voriconazole, followed by oral voriconazole tablets). Reexamination of his WBC, NEUT, hemoglobin, PLT, albumin, and globulin yielded results of $11.20 \times 10^{9} / \mathrm{L}, 76.0 \%, 72.9 \mathrm{~g} / \mathrm{L}, 280.4 \times 10^{9} / \mathrm{L}$, 


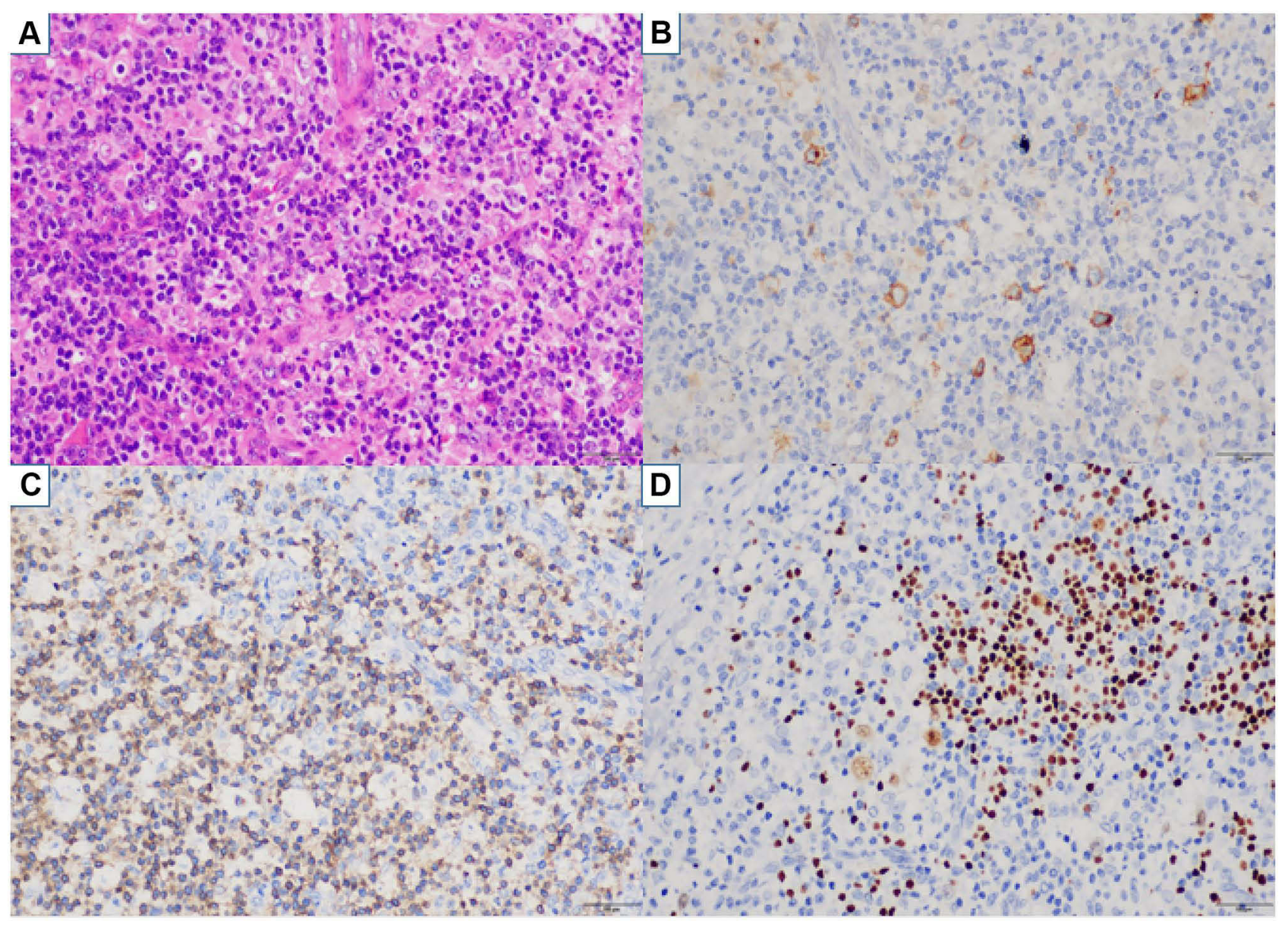

Figure 3 NSHL-related pathological results (A-D). (A) A background of complex inflammatory cells scattered with heterogeneous Hodgkin-like large cells. (B) CD30(+). (C) CD3(-). (D) PAX5( \pm ) (all magnification times are $\times 400$ ).

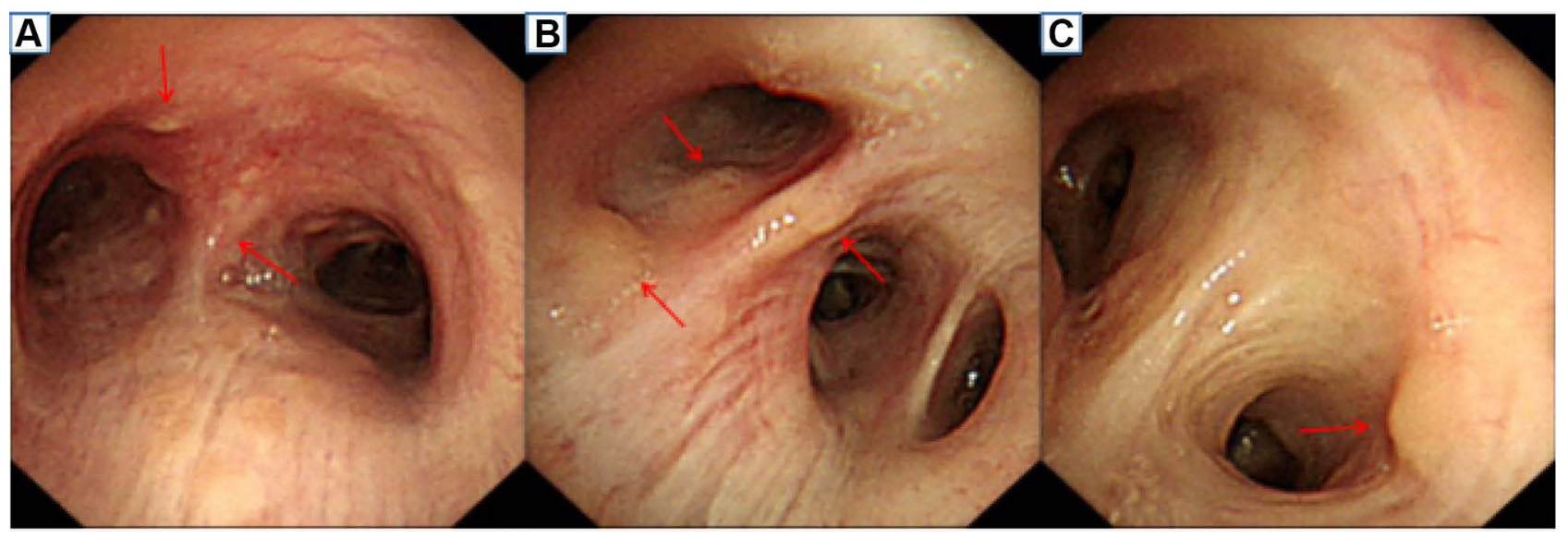

Figure 4 Bronchoscopic examinations. (A-C) The trachea and the walls of the left and right main bronchi are scattered with small nodules. The nodules are partially fused near the carina (the red arrow indicates the lesion site).

$30.7 \mathrm{~g} / \mathrm{L}$, and $40.0 \mathrm{~g} / \mathrm{L}$, respectively. A chest $\mathrm{CT}$ showed that both lung lesions and the mediastinal lymph nodes were decreased in size on February 5, 2018 (Figure 1E and F). The patient was diagnosed with NSHL (Stage II, Group B) and disseminated TSM (in the blood, lungs, skin, and subcutaneous tissue). After the patient was discharged on February 6, 2018, he continued taking voriconazole tablets orally and changed to itraconazole capsule treatment on 


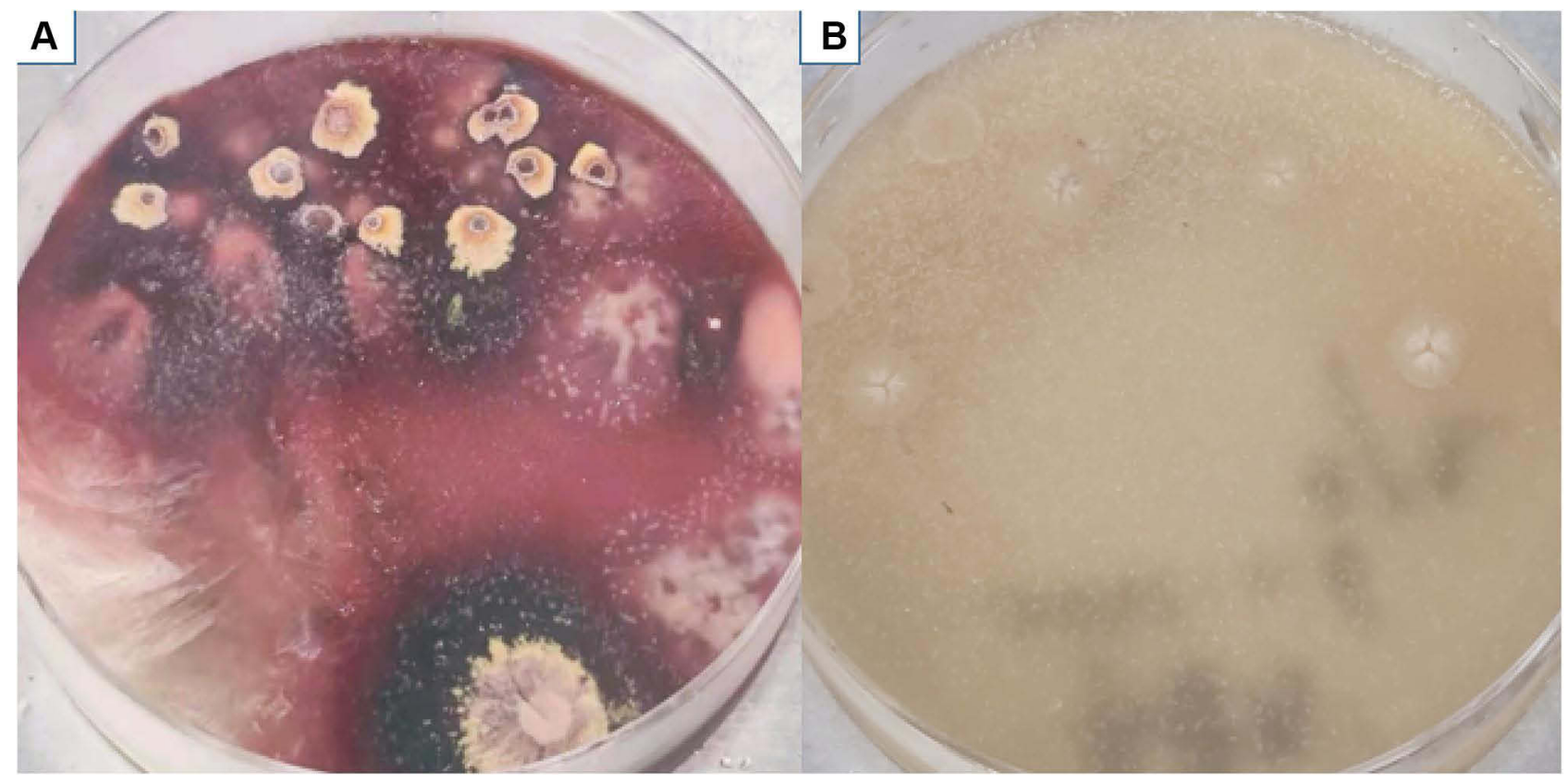

Figure 5 Microbiological examination-colony morphology (A and B). (A) T. marneffei presented in the mycelial phase, with the presence of broom branches and production of red pigment at $25^{\circ} \mathrm{C}$. (B) Yeast phase at $37^{\circ} \mathrm{C}$.

June 12, 2018. He was hospitalized for "fever" on June 29, 2018. No signs of T. marneffei relapse were found on clinical examination, blood culture, chest CT or bone marrow biopsy during hospitalization, but he insisted on oral itraconazole treatment. Finally, Salmonella was cultured in the blood of the patient, and he was diagnosed with sepsis. After treatment, the patient was discharged, but he still suffered from recurrent fever and died in September 2018.

\section{Discussion and Conclusions}

Hodgkin lymphoma is a malignant tumor originating from the lymphatic hematopoietic system. Primary lung cancer is rare and mostly secondary $(8-12 \%) .{ }^{4,5}$ The main clinical manifestations are multiple painless enlargements of lymph nodes. Nodular sclerosing Hodgkin lymphoma is the most common subtype of classical Hodgkin lymphoma, accounting for more than $70 \%$ of cases and occurring in adolescents and young adults. Nodular sclerosing Hodgkin lymphoma often manifests with local neck, axilla, supraclavicular and mediastinal lymph node enlargement, the latter of which are very common (approximately $80 \%$ ), with approximately half of these cases being mediastinal tumors ( $>10 \mathrm{~cm}$ in diameter). Inguinal lymph nodes are less involved and may also be accompanied by fever, night sweats, emaciation, other systemic symptoms and/or multisystem damage. ${ }^{6,7}$ Nodular sclerosing Hodgkin lymphoma is characterized by scattered neoplastic HRS cells with a rich cytoplasm, small nucleoli, and lobulated nuclei in a complex, sclerotic inflammatory cell background. Derived from mature B lymphocytes but missing B cell phenotypes (eg, CD19, B lymphocyte antigen CD20, B cell receptor CD22, CD79A), JAK-STAT, $\mathrm{NF}-\kappa \mathrm{B}$, and a cascade of additional signals are deregulated and continuously activated in HRS cells. ${ }^{8,9}$ Lymphoma patients have an abnormal immune status and low resistance and are prone to coinfection, which can be viral, bacterial or fungal, but infection with T. marneffei is rare.

It was previously believed that $T$. marneffe $i$ was common in HIV-positive patients. However, in recent years, it has been found that HIV-negative patients with an underlying disease are also susceptible to T. marneffei. These underlying diseases mainly include tissue diseases, malignant tumors, diabetes, organ transplantation, hormone or immunosuppressive treatment and other underlying diseases. Adult immunodeficiency syndrome caused by antiinterferon-gamma autoantibodies is also a high-risk factor for T. marneffei susceptibility. ${ }^{1}$ The clinical symptoms of TSM in HIV-negative hosts are diverse and include fever, cough, expectoration, superficial and deep lymph node enlargement, hepatosplenomegaly, subcutaneous nodules, and bone and joint infection. Laboratory tests showed decreased hemoglobin but increased WBC and CD4+ $\mathrm{T}$ cell counts. Early lung function may be normal, but patients often develop type I respiratory failure in later 
stages. ${ }^{10,11}$ Pulmonary imaging shows patchy exudative shadows, consolidation, fibroproliferative lesions, lytic destruction, pathological fractures, mediastinal lymph node enlargement, pleural effusion, pericardial effusion, cavities, diffuse nodular shadows and other extensive and diverse lesions. $^{12-14}$ In our case, the patient had fever, cough, expectoration, lymph node enlargement, and hard and fixed subcutaneous nodules on admission, and inflammatory indicators (eg, WBC, NEUT, PCT, CRP and ESR) were significantly increased. A chest CT scan showed diffuse nodular changes in both lungs. These findings are consistent with the reported TSM manifestations in HIVnegative hosts.

It is difficult to distinguish NSHL and disseminated TSM, both of which may present with fever, cough, expectoration, fatigue, weight loss, lymphadenopathy, and hepatosplenomegaly. The imaging manifestations are variable and nonspecific, and a definitive diagnosis often requires pathological biopsy or tissue culture. Lymph node biopsy in this patient confirmed NSLH, but we missed the diagnosis of disseminated TSM in the early stage. The pathological types of TSM are associated with the body's immune status. When the patient's immune status is severely compromised, the patient often has disseminated infections, and the patient's lymph node structure is completely destroyed and is often replaced by diffuse hyperplastic cells (containing abundant fungal spores), which eventually form a confluent granuloma structure and can be accompanied by incomplete caseous necrosis. When patients have a normal immune status, this disease mainly presents as a localized infection and can cause abscess development. A small number of fungal spores can be found in tissue cells on the edge of the abscess. ${ }^{15}$ T. marneffei is a thermally dimorphic pathogenic fungus. The main histomorphology includes the presence of a mulberry-shaped cell mass, dachshund-like cells and a diaphragm. It was isolated from the tissue and cultured on Sabouraud dextrose agar. At $25^{\circ} \mathrm{C}$, the mycelium grew into broom branches and produced a red pigment. Yeast growth (pathogenic phase) is observed at $37^{\circ} \mathrm{C}$. PAS staining revealed oval dachshund-like cells with a diameter of 2-8 $\mu \mathrm{m}$ and a typical capsule in the middle. ${ }^{16}$ Tissue culture is the gold standard for the diagnosis of T. marneffei. However, the disadvantage is that it takes a long time (approximately 7-10 days) and often does not produce positive results if the patient is in the incubation period. ${ }^{17}$ An increasing number of new technologies have emerged, such as polymerase chain reaction (PCR), nested
PCR, enzyme-linked immunosorbent assay (ELISA), rapid immunochromatographic testing (ICT) and metagenomic sequencing (mNGS), which can be used for early detection to confirm diagnosis. mNGS can sequence a large number of DNA molecules at the same time and can yield results in 24 to 72 hours. It integrates and analyzes all nucleic acid sequences from isolates and hosts to determine the species and abundance of microorganisms and makes diagnoses based on clinical characteristics. mNGS can be used successfully to identify infections of unknown origin, including blood, respiratory and gastrointestinal tract infections. In clinical diagnosis and treatment, mNGS can be considered for early diagnosis when T. marneffei infection is suspected. ${ }^{18}$ Bone marrow examinations of TSM patients often show active hyperplasia, and T. marneffei can be cultured when the bone marrow is involved. The histomorphological findings seen in NSHL include the presence of a large amount of fibrous tissue separating the lymph nodes into birefringent nodules of different sizes; therefore, lacunar cells and R-S cells can be found. Immunohistochemistry showed positive CD30 and CD15 and negative ALK, CD20, EMA, CD43, CD3, TIA and GrB, and the bone marrow culture was negative. $^{19}$

Lymphoma is sensitive to hormonal therapy. Clinically, we should consider the possibility of other diseases, such as infectious diseases, when hematological malignancies fail to respond to hormonal therapy or are partially improved but there is progression of other signs or symptoms The patient's lymph nodes significantly decreased in size due to the hormone therapy administered at the beginning of treatment, even though he had refused standard chemotherapy after the diagnosis was made. However, the patient still had hyperpyrexia and had no change in the subcutaneous nodules. Chest CT showed an increase in the lesion size in both lungs. A bronchoscopic examination demonstrated the presence of hyperplastic masses of the airway wall, and the pathology showed the presence of suppurative granuloma. Eventually, we cultured T. marneffei from lung tissue, although no typical fungal spores were found under a microscope. The above symptoms completely improved after the patient accepted antifungal treatment.

It is very difficult to treat disseminated TSM. The current treatment of HIV-negative TSM mainly consists of the classical treatment of HIV-positive TSM patients: amphotericin B (0.7-1.0 mg/kg/day for 2 weeks) and sequential itraconazole (200 $\mathrm{mg}$ twice daily for 10 weeks). ${ }^{20}$ 
Nevertheless, amphotericin B has significantly renal toxicity and other side effects. Relevant literature reports show that voriconazole has good clinical efficacy and safety for TSM, and the two administration methods of oral administration and intravenous infusion can be interchanged. The toxicity and side effects of voriconazole are less than those of amphotericin B and its derivatives. It can also be used as one of the first choices for the treatment of TSM. Appropriate treatment for Hodgkin lymphoma is based on accurate staging. Chemotherapy plus local radiation therapy is recommended for early Hodgkin's lymphoma, and it is now believed that more than $80 \%$ of newly diagnosed Hodgkin lymphoma patients younger than 60 years of age can achieve a permanent response and be considered cured. However, patients with advanced Hodgkin lymphoma need to undergo a long course of chemotherapy and radiotherapy, and the characteristics of group B symptoms and large tumor size $(>10 \mathrm{~cm})$ are still the most important prognostic factors of Hodgkin's lymphoma. ${ }^{21,22}$ In our case, the patient suffered from NSHL combined with a T. marneffei infection, and treatment for both was equally important. Timely diagnosis, early antifungal treatment and standard antitumor treatment can help achieve satisfactory clinical efficacy.

Clinically, we should pay attention to the differentiation of hematological system tumors, especially Hodgkin lymphoma and TSM, when we encounter patients with fever, lymph node enlargement, hepatosplenomegaly, and hematological and respiratory system involvement. We should not ignore the possibility of the coexistence of both. When the patient has multiple systemic lesions, simultaneous and accurate histopathological examinations, cultures of multiple sites or mNGS should be performed as much as possible. A PET/CT examination can be performed if the patient has sufficient financial resources, and biopsy can be performed on tissue sites with a high metabolism. ${ }^{23}$ Clinicians should observe the changes in the condition closely and dynamically and should analyze the pathogenesis comprehensively to reduce the probability of a clinical misdiagnosis or a missed diagnosis.

\section{Abbreviations}

NSHL, nodular sclerosing Hodgkin lymphoma; TSM, talaromycosis marneffei; HL, Hodgkin lymphoma; HIV, human immunodeficiency virus; WBC, white blood cell; NEUT, neutrophil ratio; PLT, platelet count; PCT, procalcitonin, CRP, C-reactive protein; ESR, erythrocyte sedimentation rate; CT, computed tomography.

\section{Data Sharing Statement}

All the data are fully available without restriction.

\section{Ethics Approval and Informed Consent}

This study was approved by the Faculty of Medicine at The First Affiliated Hospital of Guangxi Medical University to publish the case details [2021(KY-E-219)]. All patients or the patients' parents provided written informed consent for publication of this case report and any accompanying images. The study was carried out in accordance with the principles of the Declaration of Helsinki. The first author vouches for the completeness and accuracy of the data and for the adherence of the study to the protocol.

\section{Consent for Publication}

Signed consent was obtained for the publication of the case details from the participant.

\section{Acknowledgments}

We have not received substantial contributions from nonauthors.

\section{Author Contributions}

ZY made substantial contributions to the conception and design of the study; acquisition, analysis, and interpretation of the data; and drafting of the manuscript. WZ, YQ and GL participated in the analysis and interpretation of the data. JZ made substantial contributions to the conception and design of the study; acquisition, analysis, and interpretation of the data; and critical revision of the manuscript for important intellectual content and was accountable for all aspects of the work to ensure that questions related to the accuracy and integrity of any part of the work were appropriately investigated and resolved. All authors made a significant contribution to the work reported, whether that is in the conception, study design, execution, acquisition of data, analysis and interpretation, or in all these areas; took part in drafting, revising or critically reviewing the article; gave final approval of the version to be published; have agreed on the journal to which the article has been 
submitted; and agree to be accountable for all aspects of the work.

\section{Funding}

This work was supported by grants from the Natural Science Foundation of China [NSFC81760010 and 82060364] and the Science and Technology Department of Guangxi Zhuang Autonomous Foundation of Guangxi Key Research and Development Program (No. GuikeAB20238025).

\section{Disclosure}

The authors report no conflicts of interest related to this work.

\section{References}

1. Qiu Y, Feng X, Zeng W, Zhang H, Zhang J. Immunodeficiency disease spectrum in HIV-negative individuals with Talaromycosis. $J$ Clin Immunol. 2021;41(1):221-223. doi:10.1007/s10875-02000869-5

2. Mauch PM, Kalish LA, Kadin M, Coleman CN, Osteen R, Hellman S. Patterns of presentation of Hodgkin disease. Implications for etiology and pathogenesis. Cancer. 1993;71 (6):2062-2071. doi:10.1002/1097-0142(19930315)71:6<2062::AIDCNCR2820710622>3.0.CO;2-0

3. Townsend W, Linch D. Hodgkin's lymphoma in adults. Lancet. 2012;380(9844):836-847. doi:10.1016/S0140-6736(12)60035-X

4. Radin AI. Primary pulmonary Hodgkin's disease. Cancer. 1990;65 (3):550-563. doi:10.1002/1097-0142(19900201)65:3<550::AIDCNCR2820650328>3.0.CO;2-W

5. Jóna Á, Illés Á, Szemes K, Miltényi Z. Pulmonalis eltérések Hodgkin-lymphomában. Orv Hetil. 2016;157(5):163-173. doi:10.1556/650.2016.30351

6. Shanbhag S, Ambinder RF. Hodgkin lymphoma: a review and update on recent progress. CA Cancer J Clin. 2017;68(2):116-132. doi: $10.3322 /$ caac. 21438

7. Ansell SM. Hodgkin lymphoma: a 2020 update on diagnosis, risk-stratification, and management. Am J Hematol. 2020;95 (8):978-989. doi:10.1002/ajh.25856

8. Connors JM, Cozen W, Steidl C, et al. Hodgkin lymphoma. Nat Rev Dis Primers. 2020;6(1):61. doi:10.1038/s41572-020-0189-6

9. Mathas S, Hartmann S, Küppers R. Hodgkin lymphoma: pathology and biology. Semin Hematol. 2016;53(3):139-147. doi:10.1053/j. seminhematol.2016.05.007

10. Chan JF, Lau SK, Yuen KY, Woo PC. Talaromyces (Penicillium) marneffei infection in non-HIV-infected patients. Emerg Microbes Infect. 2016;5(3):e19. doi:10.1038/emi.2016.18

Infection and Drug Resistance

\section{Publish your work in this journal}

Infection and Drug Resistance is an international, peer-reviewed openaccess journal that focuses on the optimal treatment of infection (bacterial, fungal and viral) and the development and institution of preventive strategies to minimize the development and spread of resistance. The journal is specifically concerned with the epidemiology of
11. Zhang JQ, Yang ML, Zhong XN, et al. A comparative analysis of the clinical and laboratory characteristics in disseminated penicilliosis marneffei in patients with and without human immunodeficiency virus infection. Chinese journal of tuberculosis and respiratory diseases. 2008;31(10). doi:10.3321/j.issn:1001-0939.2008.10.009

12. Taramelli D, Brambilla S, Sala G, et al. Effects of iron on extracellular and intracellular growth of Penicillium marneffei. Infect Immun. 2000;68(3):1724-1726. doi:10.1128/IAI.68.3.1724-1726.2000

13. Supparatpinyo K, Chiewchanvit S, Hirunsri P, Uthammachai C, Nelson KE, Sirisanthana T. Penicillium marneffei infection in patients infected with human immunodeficiency virus. Clin Infect Dis. 1992;14(4):871-874. doi:10.1093/clinids/14.4.871

14. Deng Z, Ribas JL, Gibson DW, Connor DH. Infections caused by Penicillium marneffei in China and Southeast Asia: review of eighteen published cases and report of four more Chinese cases. Rev Infect Dis. 1988;10(3):640-652. doi:10.1093/clinids/10.3.640

15. Ma Y, He RK. Pathology of AIDS-related lymphadenopathy: a study of 18 biopsy cases. Zhonghua Bing Li Xue Za Zhi. 2005;34 (12):776-779.

16. Vanittanakom N, Cooper CR Jr, Fisher MC, Sirisanthana T. Penicillium marneffei infection and recent advances in the epidemiology and molecular biology aspects. Clin Microbiol Rev. 2006;19 (1):95-110. doi:10.1128/CMR.19.1.95-110.2006

17. Chen X, Jia L, Wu Y, et al. A mass in the upper abdomen derived from Talaromyces marneffei infected lymphadenopathy: a case report. BMC Infect Dis. 2021;21(1):750. doi:10.1186/s12879-02106489-7

18. Chen Q, Qiu Y, Zeng W, Wei X, Zhang J. Metagenomic next-generation sequencing for the early diagnosis of talaromycosis in HIVuninfected patients: five cases report. BMC Infect Dis. 2021;21:865. doi:10.1186/s12879-021-06551-4

19. Zhang O. Pathological diagnosis of typical Hodgkin's lymphoma. World Health Dig Med Period. 2011:13-0178-01. Available from: https://d.wanfangdata.com.cn/periodical/Ch1QZXJ pb2RpY2FsQ0hJTmV3UzIwMjExMTMwEg96d2prd3oyMDExMT MxNTYaCDRmcmphOWU2. Accessed December 21, 2021.

20. Supparatpinyo K, Perriens J, Nelson KE, Sirisanthana T. A controlled trial of itraconazole to prevent relapse of Penicillium marneffei infection in patients infected with the human immunodeficiency virus. $N$ Engl $J$ Med,1998;339(24):1739-1743. doi: 10.1056/NEJM199812103392403.

21. Eichenauer DA, Engert A, André M, et al. Hodgkin's lymphoma: ESMO clinical practice guidelines for diagnosis, treatment and follow-up. Ann Oncol. 2014;25(Suppl 3):iii70-75. doi:10.1093/annonc/ mdu181

22. Connors JM. State-of-The-art therapeutics: Hodgkin's lymphoma. J Clin Oncol. 2005;23(26):6400-6408. doi:10.1200/JCO.2005.05.016

23. Chen D, Chang C, Chen M, et al. Unusual disseminated Talaromyces marneffei infection mimicking lymphoma in a non-immunosuppressed patient in East China: a case report and review of the literature. $B M C$ Infect Dis. 2020;20(1):800. doi:10.1186/s12879-020-05526-1 antibiotic resistance and the mechanisms of resistance development and diffusion in both hospitals and the community. The manuscript management system is completely online and includes a very quick and fair peerreview system, which is all easy to use. Visit http://www.dovepress.com/ testimonials.php to read real quotes from published authors. 